\title{
BMJ Open A cluster randomised controlled trial to determine the effect of community mobilisation and advocacy on men's use of violence in periurban South Africa: study protocol
}

Nicola J Christofides, ${ }^{1}$ Abigail M Hatcher, ${ }^{1,2}$ Angelica Pino, ${ }^{3}$ Dumisani Rebombo, ${ }^{3}$ Ruari Santiago McBride, ${ }^{1}$ Althea Anderson, ${ }^{3}$ Dean Peacock ${ }^{3}$

To cite: Christofides NJ, Hatcher AM, Pino A, et al. A cluster randomised controlled trial to determine the effect of community mobilisation and advocacy on men's use of violence in periurban South Africa: study protocol. BMJ Open 2018;8:e017579. doi:10.1136/ bmjopen-2017-017579

- Prepublication history for this paper is available online. To view these files, please visit the journal online (http://dx.doi org/10.1136/bmjopen-2017017579).

Received 1 May 2017

Revised 3 January 2018

Accepted 5 January 2018
Check for updates

${ }^{1}$ Faculty of Health Sciences, School of Public Health, University of the Witwatersrand, Johannesburg, South Africa ${ }^{2}$ Department of Medicine, University of California, San Francisco, San Francisco, CA, USA

${ }^{3}$ Sonke Gender Justice, Johannesburg, South Africa

Correspondence to Dr Nicola J Christofides; nicola.christofides@wits.ac.za

\section{ABSTRACT}

Objective This paper describes the design and methods of a cluster randomised controlled trial (C-RCT) to determine the effectiveness of a community mobilisation intervention that is designed to reduce the perpetration of violence against women (VAW).

Methods and analysis A C-RCT of nine intervention and nine control clusters is being carried out in a periurban, semiformal settlement near Johannesburg, South Africa, between 2016 and 2018. A community mobilisation and advocacy intervention, called Sonke CHANGE is being implemented over 18 months. It comprises local advocacy and group activities to engage community members to challenge harmful gender norms and reduce VAW. The intervention is hypothesised to improve equitable masculinities, reduce alcohol use and ultimately, to reduce VAW. Intervention effectiveness will be determined through an audio computer-assisted self-interview questionnaire with behavioural measures among 2600 men aged between 18 and 40 years at baseline, 12 months and 24 months. The primary trial outcome is men's use of physical and/or sexual VAW. Secondary outcomes include harmful alcohol use, gender attitudes, controlling behaviours, transactional sex and social cohesion. The main analysis will be intention-totreat based on the randomisation of clusters. A qualitative process evaluation is being conducted alongside the C-RCT. Implementers and men participating in the intervention will be interviewed longitudinally over the period of intervention implementation and observations of the workshops and other intervention activities are being carried out

Ethics and dissemination Ethical approval was obtained from the University of the Witwatersrand Human Research Ethics Committee and procedures comply with ethical recommendations of the United Nations Multi-Country Study on Men and Violence. Dissemination of research findings will take place with local stakeholders and through peer-reviewed publications, with data available on request or after 5 years of trial completion.

Trial registration number NCT02823288; Pre-result.

\section{INTRODUCTION}

Violence against women (VAW), including sexual and/or physical violence, is a leading
Strengths and limitations of this study

- There is limited evidence from low-income and middle-income countries of how best to prevent men's use of violence against women (VAW).

- Strengths include randomisation of clusters after baseline data collection and intention-to-treat analysis.

- Limitations include risk of contamination across clusters and potential lost to follow-up of men over 2 years.

cause of morbidity and mortality among the $35 \%$ of women globally who experience it. ${ }^{2}$ Prevalence of intimate partner and non-partner VAW is high in Southern Africa. Large studies among South African men found that $27.5 \%-31.8 \%$ report enacting physical and/or sexual violence towards partners, ${ }^{3}$ and $27.6 \%$ of men have ever raped. ${ }^{4}$ These high rates of violence against partners and non-partners are consistent with population-based findings from studies among men in other regions globally. ${ }^{56}$

There is a growing consensus that hegemonic masculinities lead to harmful health behaviours, including VAW. ${ }^{7}$ Research suggests that men who strictly adhere to dominant norms of masculinity (eg, toughness, virility, power) are more likely to perpetrate VAW. ${ }^{6}{ }^{8}$ However, the evidence base for precisely how interventions can encourage men to reconstruct masculinities and whether this would result in a reduction of perpetration of VAW is limited. Much of the literature focuses on the problems of masculinity, ${ }^{9}$ and evidence from existing programmes is restricted to a handful of small interventions. ${ }^{10} 11$ In South Africa, two trials with primary outcomes that aimed 
to reduce the incidence of HIV had some promising results at reducing VAW. The IMAGE trial combined economic intervention with gender training workshops and reported a reduction in women's reports of pastyear VAW by $51 \% .^{12}$ Stepping Stones, a series of community-based workshops with women and men, showed a $38 \%$ reduction in men's perpetration of violence after 2 years of follow-up. ${ }^{10}$

Sonke Gender Justice (Sonke), a South African non-governmental organisation, has been running gender transformative, community-based programmes since 2006. The core Sonke intervention has evolved over more than 10 years and is premised on mobilising communities to take action against VAW. The activities include a series of group workshops and other reflective activities to challenge harmful gender norms and educate men about gender-based violence and HIV risks. ${ }^{13} 14$ The theory underpinning the intervention is that through community outreach and advocacy, harmful values and practices can be transformed towards gender equity and thereby reduce VAW. Equitable masculine norms manifest through behaviours and attitudes that are considered to reduce the likelihood of VAW (eg, equality, respect, intimacy, responsibility). ${ }^{1516}$ The Sonke Community Health Action for Norms and Gender Equity (CHANGE) intervention adds to existing Sonke activities by bolstering community action and local advocacy specifically around men's use of VAW. CHANGE and posits that masculine norms can be progressively transformed through community activities that stimulate personal as well as collective reflection and action.

This type of gender transformative intervention is under-researched, ${ }^{17}$ but there is preliminary qualitative evidence though that such an approach is promising. ${ }^{18} 19$ In order to reach global goals of eliminating VAW, ${ }^{20}$ it is crucial to understand how multilevel programming may impact men's use of violence. The aim of the cluster randomised controlled trial (C-RCT) is to determine the effectiveness of the Sonke CHANGE intervention to prevent men's use of sexual and or physical violence against an intimate partner and reduce the severity of perpetration by men aged 18-40 years living in a periurban South African settlement over 2 years of follow-up.

\section{METHODOLOGY}

This trial is funded by the UK Agency for International Development (UKAID) through What Works to Prevent Violence, a global consortium of research managed by the South African Medical Research Council. What Works had broad input on the scientific and ethical considerations of study design, and has an advisory role in data collection, management, analysis and interpretation of data. The writing and submission of the report is the decision of the investigative team.

The Consolidated Standards of Reporting Trials and Standard Protocol Items for Randomised Trials guidelines have been followed, and the study protocol adheres to the Standard Protocol Items: Recommendations for Interventional Trials.

\section{Participants, interventions and outcomes}

The trial is being conducted in a semiformal 'township' located near Johannesburg, South Africa. The periurban settlement took form in the mid-1990s, when the fall of apartheid 'pass laws' allowed non-whites to move closer to cities to seek employment. Most residents live in government-subsidised housing and informal tin shacks. Few exact population estimates exist, but most assume the 'township' is now home to between 250000 and a half million people, including high numbers of migrants from other African countries. Many residents lack access to basic services such as running water, sewerage and rubbish removal. Citizen officials estimate that half the population in the settlement is unemployed. ${ }^{21}$

Recruitment of participants was led by the trial team of trained research assistants. Men who lived in the area for at least 12 months and were 18-40 years old were eligible to be recruited. Men over the age of 40 years are not being prevented from participating in community mobilisation or any of the Sonke CHANGE intervention activities but were not be eligible to be recruited for the trial. The study is described as a project about men's lives and relationships, rather than about violence, to prevent undue stigma for study participation. ${ }^{22}$

\section{Trial design}

A two-arm C-RCT is being conducted as shown in figure 1. Due to the informality of geographical boundaries within the periurban settlement, a cluster is defined as a neighbourhood bordered by a community landmark such as a church, community hall or communal water source. These landmarks were mapped through transect walks using global positioning systems coordinates obtained on a Samsung Tablet application Map Coordinates. The 18 clusters, identified for the purposes of the trial, were evenly spaced throughout the community and contained dwellings falling within a radius of $0.4 \mathrm{~km}$ of each community landmark.

Clusters identified for inclusion in the study are not contiguous and each is bordered by a natural boundary (such as a stream) or by a sizeable physical distance of at least $400 \mathrm{~m}$. While contamination is a concern, spillover effects in this type of C-RCT cannot be perfectly contained. Any intentional or unintentional contamination is being measured through a series of items on the questionnaire that determine participant exposure to specific intervention components. This data will be triangulated with qualitative process evaluation data to provide a contextualised understanding of contamination/spillover effects.

\section{Intervention activities}

The Sonke CHANGE Intervention is being implemented over a period of 18 months (April 2016 to November 2017). Sonke Gender Justice is implementing a multilevel approach to stimulate critical reflection among men and 


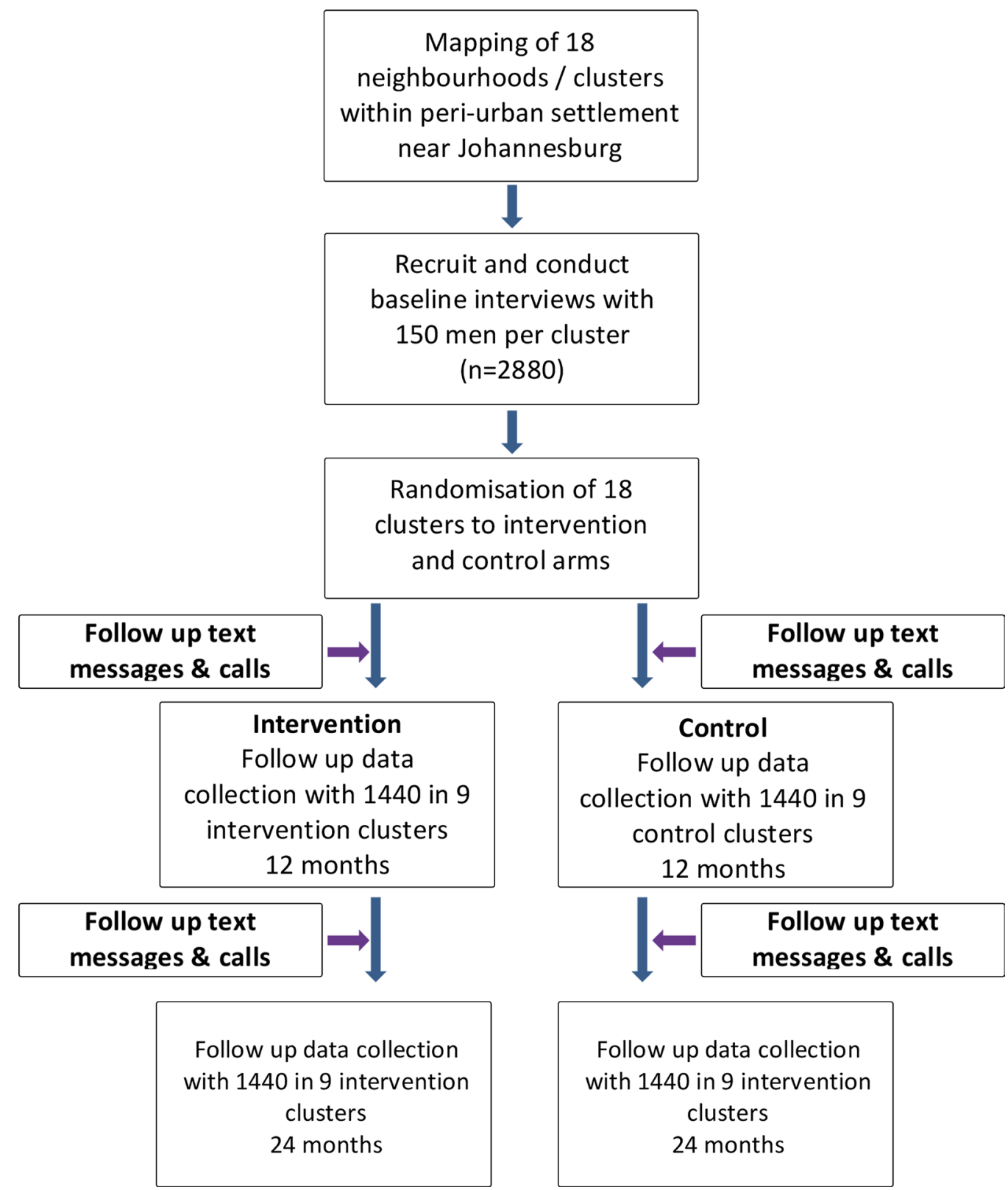

Figure 1 Flow diagram showing trial recruitment and follow-up at 12 and 24 months.

promote equitable gender norms and non-violent masculine attitudes and practices. The Sonke core intervention staff comprises a full-time manager and six community mobilisers (three men and three women) recruited from the community where the study is taking place. Two community mobilisers are responsible for three intervention clusters. Intervention activities comprised workshops initially run by community mobilisers, mobilisation led by Community Action Teams (CATs) and advocacy (see table 1). Community mobilisers received extensive training over several months, comprised of a manualised curriculum that includes participatory activities, values clarification and shadowing established mobilisers working in a different community.

Workshops aim to challenge inequitable and harmful ideas about manhood and encourage men to take action to promote equality. ${ }^{23} 24$ They draw on Freirean education paedagogy and principles and promote reflection and a commitment to action. ${ }^{25}$ A dedicated workshop curriculum was developed specific to the goals of the Sonke CHANGE intervention, with additional materials created to bolster emphasis on VAW prevention.

CATs comprised men and women who mobilise community members on a voluntary basis around issues of gender transformation. CATs are recruited through workshops that are run by community mobilisers. Participants who are particularly interested in the content of the workshops are invited to join a CAT. In practice, CAT members include approximately 20-40 members of the local community, all of whom live in intervention clusters. The process of recruiting and training CAT members occurs on an ongoing basis, depending on retention and planned mobilisation activities. CATs are trained through week-long, manualised workshops that are led 
Table 1 Sonke CHANGE intervention activities

\begin{tabular}{|c|c|c|}
\hline Activity & Frequency & $\begin{array}{l}\text { Target people reached } \\
\text { per cluster, per activity }\end{array}$ \\
\hline \multicolumn{3}{|l|}{ CHANGE training } \\
\hline Recruit potential Community Action Team (CAT) members & Ongoing as needed & 15 \\
\hline 5-day training & Once off for CAT members & 15 \\
\hline $\begin{array}{l}\text { Individual commitment to action and report back (community } \\
\text { bystander activities) }\end{array}$ & Monthly & 5 \\
\hline Refresher training & Quarterly & 12 \\
\hline \multicolumn{3}{|l|}{ CAT community mobilisation } \\
\hline Door-to-door campaign & $2 x$ week & 60 \\
\hline Street intervention (banner/poster discussion) & 2x week & 10 \\
\hline CHANGE workshops-2-day training & $2 \times$ month & 30 \\
\hline $\begin{array}{l}\text { Mini-workshops ( } 1-2 \text { hours) held in local taverns, churches, } \\
\text { schools }\end{array}$ & Weekly & 12 \\
\hline Digital stories film screenings & $2 x$ month & 50 \\
\hline Mural paintings & $2 x$ month & 80 \\
\hline Ambush theatre & Monthly & 50 \\
\hline Community dialogues & Monthly & 80 \\
\hline Debate session (at schools) - community mobiliser & Monthly & 30 \\
\hline Most significant change story & Monthly (start at 6 months) & 1 \\
\hline $\begin{array}{l}\text { Stakeholder meeting (local organisations, community police } \\
\text { forums, community leaders) }\end{array}$ & $2 x$ week & 80 \\
\hline Street soccer-violence against women information or debate & Quarterly & 15 \\
\hline Open houses to discuss a topic or theme & Quarterly & 60 \\
\hline Training local organisations (3 days) & Annually & 30 \\
\hline \multicolumn{3}{|l|}{ Advocacy } \\
\hline Lobbying & Ongoing & as needed \\
\hline Marching/protest & Ongoing & as needed \\
\hline Media advocacy & Ongoing & as needed \\
\hline
\end{tabular}

by Sonke community mobilisers. Following training and a process of shadowing the community mobilisers (lasting between 1 and 6 months, depending on the skills of the CAT members), CATs initiate a number of activities throughout all nine intervention clusters, such as workshops, ambush theatre (spontaneous theatre that occurs on the street), door-to-door educational outreach and community dialogues. CAT activities aim to reach a large number of people in each community to achieve 'saturation' of new ideas and social norms. CATs receive transportation reimbursement but do not receive a salary for their efforts.

Advocacy is undertaken by Sonke staff, including community mobilisers, who aim to hold government and other duty bearers to account for VAW prevention. Sonke staff join local community structures such as community policing forums, school governing bodies, hospital committees, church groups, and football clubs and use their presence to advance community education and local government accountability.
Workshops address hegemonic masculinities on the personal level; CATs address hegemonic masculinity norms at a community level and advocacy addresses hegemonic masculinity on the level of governance. Together this multilevel approach intends to stimulate critical reflection at the individual, social and political levels.

In the control cluster, communities receive the standard care. This choice of comparator is deemed ethical since little evidence exists for the efficacy and safety of the intervention being tested. Any pre-existing interventions or community-based activities are continuing. However, communities in the control arm are not being intentionally exposed to Sonke CHANGE intervention activities. One caveat is that advocacy may necessarily overlap across cluster boundaries, since it is likely to engage large parts of the periurban community. This scientific limitation will be accounted for during follow-up data collection, which asks individuals about their exposure to Sonke advocacy. 


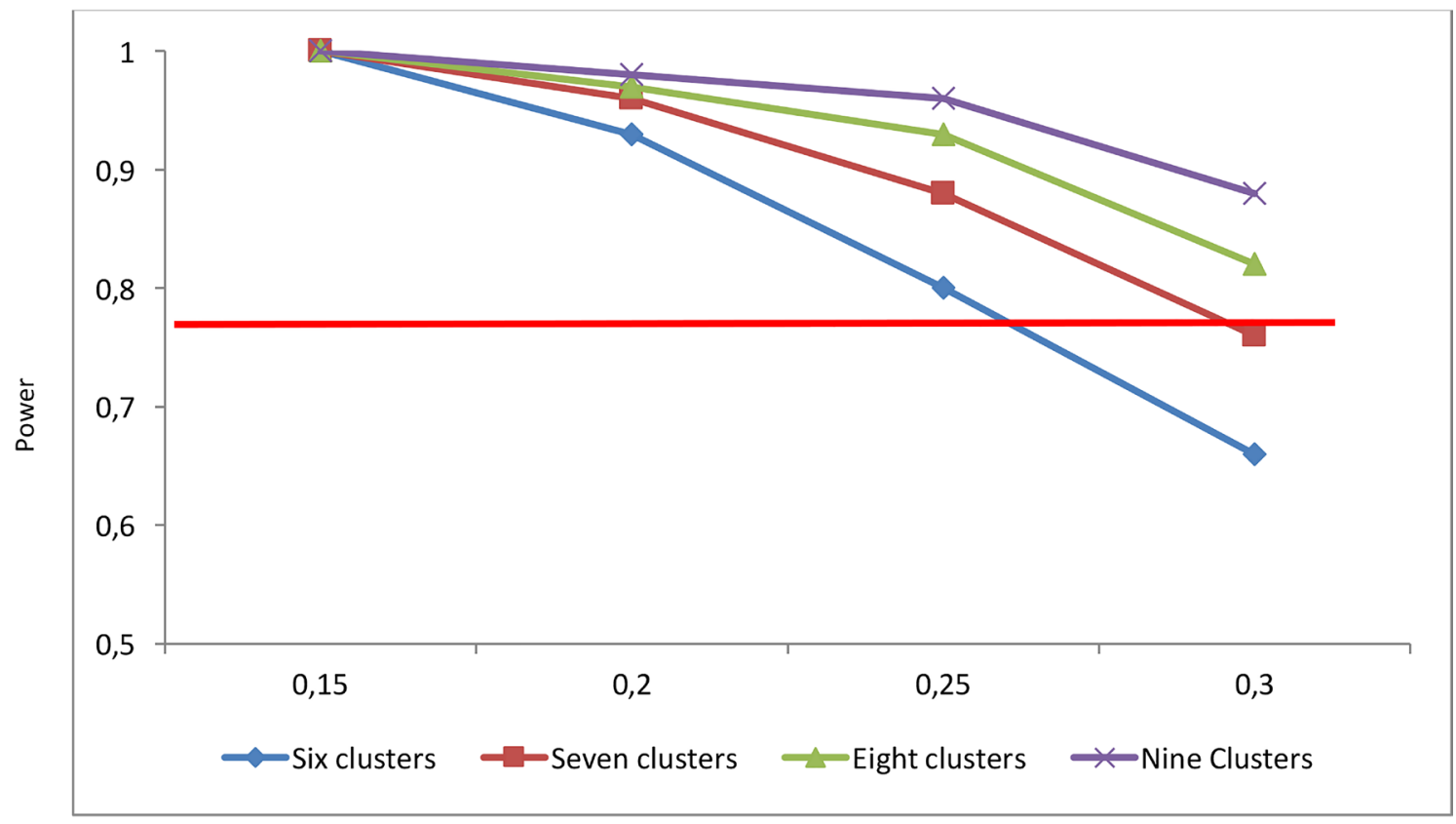

Coefficient of variation

Figure 2 Power calculation showing a reduction in the prevalence of men's use of intimate partner violence in the previous 12 months from $12 \%$ to $7 \%$ with six, seven, eight or nine clusters per arm and approximately 150 men per cluster.

\section{Outcome measures}

The long-term goal of the intervention is to reduce men's use of intimate partner and non-partner VAW. A number of primary and secondary measures have been defined a priori.

\section{Primary outcome measure: men's reported violence}

Men's use of violence towards an intimate partner is measured using an adapted version of the questionnaire from the South African Medical Research Council's Study on Men's Health and Relationships. ${ }^{627}$ The questionnaire includes items around emotional abuse, economic abuse, physical violence and sexual violence. Primary outcomes are defined as dichotomous outcomes: any use of physical violence and/or any use of sexual violence against a partner in the past 12 months. The severity of sexual and/ or physical violence use will use the Likert scale responses to violence items. ${ }^{28}$

\section{Secondary outcome measures}

Harmful alcohol use is measured using the Alcohol Use Disorders Identification Test, a 10-item scale designed to measure alcohol consumption and identify risks for alcohol abuse and dependence. ${ }^{29}$

Perpetration of non-partner rape measured using an adapted version of the questionnaire from the South African Medical Research Council's Study on Men's Health and Relationships. ${ }^{627}$

Gender attitudes are measured using the Gender Equitable Men's Scale ${ }^{30}$ and the Gender Norms scale on whether a man perceives that his community holds those beliefs. $^{31}$
Male controlling behaviour is measured using the Sexual Relationship Power and Control scale items. ${ }^{32}$ This scale has been validated in South Africa, ${ }^{33}$ and has been used by members of our team in previous studies. ${ }^{34}$

Parenting is measured by the Parent-Child Conflict Tactics Scale, a series of items about parental psychological abuse and physical discipline of children. ${ }^{35}$

Transactional sex is measured using the Medical Research Council's standard measure for South Africa. This measures transactional sex among casual partners. ${ }^{31}$

Social cohesion is assessed using a measure from the Stepping Stones questionnaire. ${ }^{10}$

Mental health is measured using multiple scales. Depression is measured using the Center for Epidemiological Studies Depression (CES-D) tool, a brief, validated instrument based on the nine diagnostic criteria for Diagnostic and Statistical Manual of Mental Disorders, 4th Edition depressive disorders. ${ }^{36}$ The Harvard Trauma Questionnaire is a cross-cultural instrument for measuring symptoms associated with post-traumatic stress disorder. ${ }^{37}$

\section{Covariates}

Partnership characteristics include basic demographics about sexual partners and sexual behaviour from the Stepping Stones questionnaire. ${ }^{10}$

Socioeconomic status is assessed using items from the United Nations Multi-Country Study around education, marital status, household size and monthly income.

Food security is measured using the Household Hunger Scale, a three-item measure developed by the US Agency for International Development-funded Food and Nutrition Technical Assistance Project. ${ }^{38}$ 


\begin{tabular}{|c|c|c|c|c|c|c|c|}
\hline & \multicolumn{7}{|c|}{ STUDY PERIOD: January 2016-July 2018} \\
\hline & \multirow{2}{*}{$\begin{array}{c}\text { Enrolment } \\
\begin{array}{c}\text { Feb-April } \\
2016\end{array}\end{array}$} & \multirow{2}{*}{$\begin{array}{l}\text { Allocation } \\
\text { April } 2016\end{array}$} & \multicolumn{4}{|c|}{ Post-allocation } & \multirow{2}{*}{$\begin{array}{c}\begin{array}{c}\text { Close- } \\
\text { out }\end{array} \\
\text { July } 2018\end{array}$} \\
\hline TIMEPOINT & & & $\begin{array}{l}\text { May- } \\
\text { Dec } \\
2016\end{array}$ & $\begin{array}{l}\text { Feb-Jul } \\
2017\end{array}$ & $\begin{array}{l}\text { Aug- } \\
\text { Dec } \\
2017\end{array}$ & $\begin{array}{l}\text { Jan-Jun } \\
2018\end{array}$ & \\
\hline \multicolumn{8}{|l|}{ ENROLMENT: } \\
\hline \multirow{2}{*}{$\begin{array}{l}\text { Eligibility screen } \\
\text { Informed consent }\end{array}$} & $\mathrm{x}$ & & & & & & \\
\hline & $\mathrm{x}$ & & & & & & \\
\hline Allocation & & $\mathrm{x}$ & & & & & \\
\hline \multicolumn{8}{|l|}{ INTERVENTIONS: } \\
\hline \multicolumn{8}{|l|}{ [Sonke Intervention] } \\
\hline \multicolumn{8}{|l|}{ [Control/standard care] } \\
\hline \multicolumn{8}{|l|}{ ASSESSMENTS: } \\
\hline $\begin{array}{r}\text { [Date of birth, education, housing, } \\
\text { food security, income, childhood } \\
\text { trauma questionnaire] }\end{array}$ & $x$ & & & & & & \\
\hline $\begin{array}{r}\text { [Use of sexual and/or physical } \\
\text { violence] }\end{array}$ & $x$ & & & $x$ & & $x$ & \\
\hline $\begin{array}{l}\text { [Alcohol use, gender attitudes, } \\
\text { male controlling behavior, } \\
\text { parenting, social cohesion] }\end{array}$ & $x$ & & & $x$ & & $x$ & \\
\hline $\begin{array}{r}\text { [Partnership characteristics, drug } \\
\text { use, depression, PTSD] }\end{array}$ & $\mathrm{x}$ & & & $\mathrm{x}$ & & $x$ & \\
\hline
\end{tabular}

Figure 3 Schedule of enrolment, intervention and assessments for the Sonke CHANGE trial. PTSD, post-traumatic stress disorder.

Drug use is measured using a single question from the United Nations Multi-Country Study around past-year use: 'How many times have you used drugs in the last 12 months?'

Participant views and participation in violence-related campaigns is assessed using items from the Gender Links Survey. ${ }^{31}$ Exposure to the intervention prior to baseline and in both intervention and control communities are being measured through a series of questions that ask about awareness of Sonke Gender Justice, participation in workshops and other activities.

\section{Power estimates}

Little data are available to estimate incidence of men's use of VAW in South Africa. However, one population-based study that used a representative sample by Gender Links in Gauteng Province provides a point estimate of past-year use of violence among men. In the Gender Links study, $12 \%$ of men used physical or sexual violence towards a partner in the past 12 months. ${ }^{31}$ Thus, based on $12 \%$ past-year prevalence, we can estimate the study's power to detect a $5 \%$ difference if VAW decreases to $7 \%$. The power calculation is based on 150 participants per cluster in 18 clusters. A $20 \%$ adjustment for potential lost to follow-up increases to 180 the total number of men to be recruited in each cluster with a total sample size of 2880 . Figure 2 shows the power calculations based on Hayes and Moulton for six, seven, eight and nine clusters per arm with a coefficient of variation (k) ranging between $5 \%$ and $50 \% .{ }^{39}$ Data will be collected at three time points: baseline, 12 months and 24 months.

\section{Assignment of intervention}

Randomisation of clusters into the intervention or control arm was undertaken after the baseline data collection was completed. See figure 3 for the timing of allocation and assessments.

All cluster names were printed on equal sized pieces of paper and the randomisation was performed at a public event. The event was held with local leadership, trial researchers and Sonke staff in a public setting to ensure randomisation is transparent to the community. Each local leader chose one cluster name from a bag until nine clusters were allocated to the intervention arm. Clusters cannot be blinded to their study arm allocation after the 
initial data collection, nor can intervention implementers be blinded to arm allocation.

\section{Participant enrolment}

Study enrolment was initiated through a series of community meetings held in each cluster and door-to-door recruitment of men by trial staff. Men in the 18 clusters were invited to take part in a written informed consent process and thereafter asked to complete a Locator Form by a trained field worker. The Locator Form is the primary method of participant retention, and has information about the participant's dwelling and phone numbers. Locator Form data are stored separately from any other participant data to ensure confidentiality.

\section{Data collection, management and analysis}

Data collection occurs in private, confidential locations such as a community hall, or yard identified in each cluster. Data collection is facilitated by trained interviewers, and conducted in the language of participant choice (English, isiZulu, Tsonga or Sepedi). Interviewers are using an electronic data system called Open Data Kit on 7-inch Samsung tablet computers that operate on the Android platform. These tablet computers are inexpensive and easy to carry, and allow ease of data collection. Electronic data collection provides a standardised method that minimises user bias and improves data quality as it precludes data entry of paper forms. Security of data can be improved through use of electronic data collection (vs using paper forms), since data are uploaded to an encrypted server at the end of each day. The server is housed at the university and has been purpose built for this study.

We are using audio computer-assisted self-interview (ACASI) software, since questions around violence can be sensitive and it is ethically challenging to handle disclosure. ${ }^{40}$ Use of ACASI prevents complex ethical issues because no interviewer or researcher can examine responses to illegal questions until the data is de-identified. This inability to see individual data is important for questions around rape and physical or sexual mistreatment of children, since South African law requires mandatory reporting of these types of criminal activities. ACASI allows important data to be collected about legal and illegal activity while ensuring anonymity and confidentiality. Of note, the additional anonymity of ACASI may also lead to more accurate reporting of VAW by men since there would be no social desirability bias typically associated with interviewer-administered questionnaires.

\section{Community advisory board}

Prior to starting data collection, the team set up a community advisory board ( $\mathrm{CAB}$ ) comprising local leadership. The members include non-governmental organisations, local residents and ward councillors (local political representatives). Once sensitised to the trial and intervention, the $\mathrm{CAB}$ introduced the study, the intervention, the ethical considerations of participating and the intended outcomes to people in the community. This serves as an opportunity to set expectations around reporting back findings to the community.

\section{Data management and statistical analyses}

Data from the baseline interviews and follow-up interview data will be abstracted from Open Data Kit databases built specifically for this study. Procedures to promote data quality include range and logical checks built into Open Data Kit and running additional error checks after data abstraction.

The main analysis will be intention-to-treat based on the randomisation of clusters. The period prevalence of violence perpetration over 24 months of follow-up will be calculated. Men's use of physical and/or sexual intimate partner violence (IPV) over the previous 12 months among the intervention and control clusters will be compared as the primary trial outcome.

Since allocation to the intervention or control arms was by cluster, all statistical assessments of variability will use the cluster as the unit of analysis. Adjusted proportions of men reporting sexual and or physical IPV perpetration in the intervention group relative to the control group will be compared, by comparison of observed and expected prevalence in each cluster. Covariates in the model will include cluster prevalence (calculated using cluster means) of men's use of IPV at baseline, sociodemographic characteristics, relationship characteristics, mental health measures and attitudinal variables.

Analyses for other primary and secondary outcomes will proceed similarly, with appropriate choices of model for outcome type. A sensitivity analysis will be conducted using individual-level data with cluster as a random effect, generalised linear mixed model correcting for small number of clusters and adjusting for baseline variables such as IPV. We will also make preliminary assessments of degree of mediation in models for primary outcomes via inclusion of mediating factors, with assessment of direct and indirect intervention effects of key mediating variables. ${ }^{41}$

Additional analyses will focus on assessing the effects of the intervention on mediating factors such as harmful alcohol use, partner communication and collective efficacy as indicated in the intervention theory of change (see figure 4). Analyses for mediating variables will either treat scores as continuous measures or categorise them according to clinical cut-offs. Initial comparisons will be based on group-specific descriptive summaries of observed outcomes and tests comparing outcomes between groups (t-tests for parametric or Mann-Whitney U test/Wilcoxon signed-rank test for non-parametric data; $\chi^{2}$ for categorical data). We will also use multivariable models regression methods to compare outcomes between groups while controlling for baseline characteristics.

\section{Process evaluation}

A process evaluation employs a research design that is qualitative and longitudinal over the period of the trial 

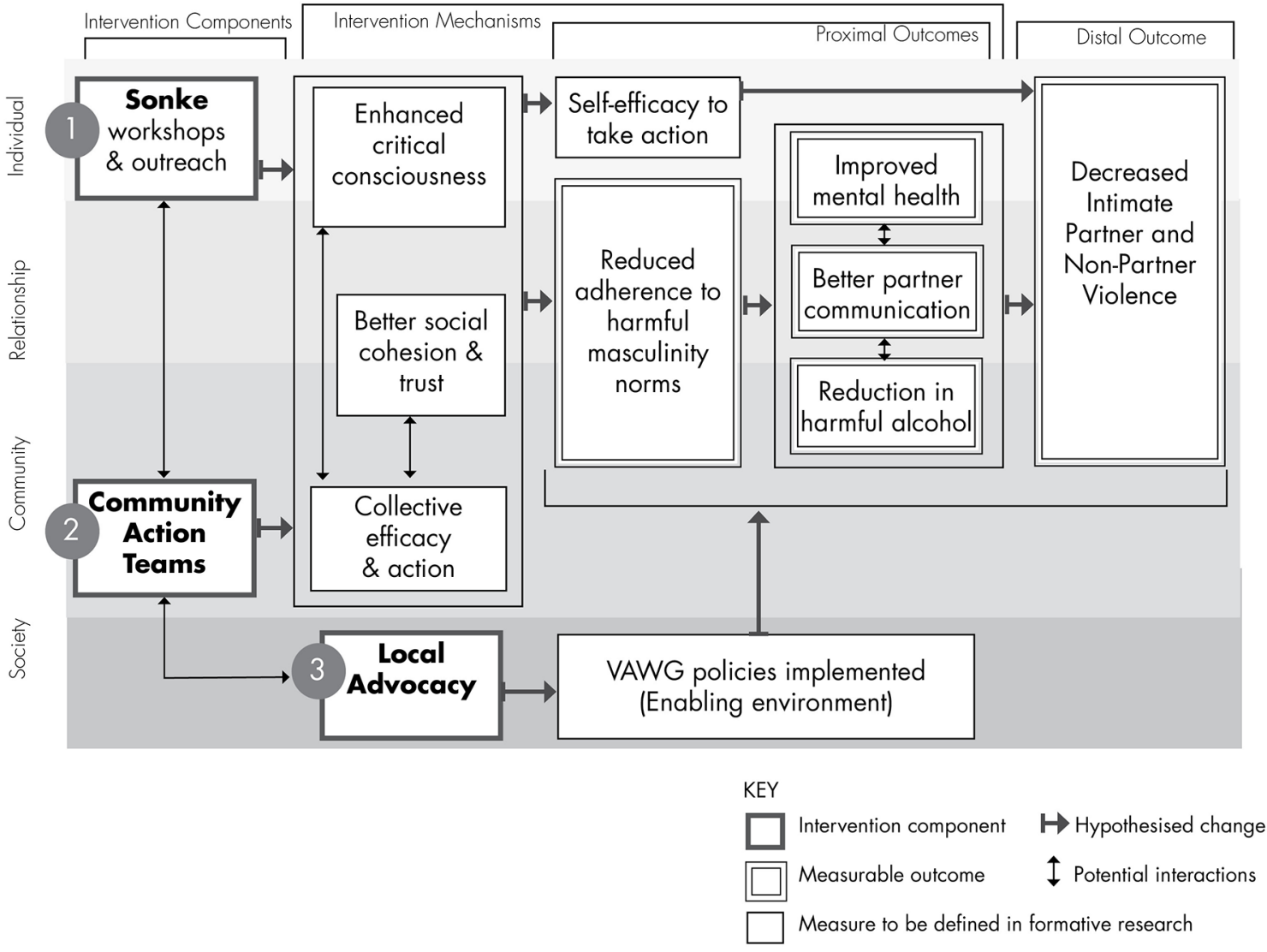

Figure 4 Sonke CHANGE trial theory of change. VAWG, violence against women and girls.

implementation, 2016-2018. It is designed to collect data that enables rich description and captures the subjective experiences of people involved in the Sonke CHANGE intervention as the intervention unfolds over time.

\section{Data collection}

A range of data collection techniques is being used for the process evaluation. In-depth interviews are conducted with stakeholders (Sonke managers $(n=5)$, trial investigators $(\mathrm{n}=3)$ and community leaders $(\mathrm{n}=5)$ ); implementers (mobilisers $(n=5)$, CAT members $(n=5)$ and fieldworkers $(\mathrm{n}=5))$, and research participants $(\mathrm{n}=10)$. In total, 38 participants are being interviewed using a semistructured topic guide. Participants are asked questions regarding the intervention implementation, contextual factors that may shape primary and secondary outcomes, and experiences in the intervention.

Maximum variation sampling is used in order to ensure a wide range of perspectives are represented among stakeholders, implementers and participants. ${ }^{42}$ This enables the collection of data that provides insights from different perspectives and enables analysis of common themes and divergent opinions across groups of actors.

Over the course of the Sonke CHANGE intervention, each of the 38 interviewees is being interviewed on multiple occasions: stakeholders two times and implementers and participants on three occasions. In total 101 interviews will be conducted. The collection of longitudinal interview data will enable analysis of shifts in perspectives and insights into how transformation might occur through participation in the intervention.

Participant observation is collected in a semistructured manner by a process evaluation researcher with expertise in ethnographic methods. The researcher is purposively attending at least one of each type of intervention activity. Participant observation will ensure unanticipated developments in the intervention are captured (eg, an unplanned intervention activity). Participant observation data will provide insight into the contextual factors that impede and facilitate the implementation of the Sonke CHANGE trial.

\section{Data analysis}

Analysis of process evaluation data will be iterative and will be managed using qualitative software. Content analysis will be used to describe the processes of participant behavioural change over time in order to determine what kinds of changes occur in men participating in intervention activities. A secondary focus will be placed on analysing theoretical themes that are identified across, and between, the qualitative data set in order to explore how and why identified changes in perceptions, beliefs or behaviour occur. A final focus will be placed on interpreting findings in order to explain the nature and meaning of changes in perception, belief or behaviour as well as to further theory development and determine the transferability of the study's findings to other contexts. 


\section{ETHICS AND DISSEMINATION}

Ethical approval was obtained from the University of the Witwatersrand Human Research Ethics Committee. Changes to the protocol are submitted to this body, and the funder is made aware of relevant amendment approvals after they are obtained.

Researchers received intensive training on VAW, the study protocol, collecting sensitive information, and ensuring data quality and participant confidentiality. Informed consent procedures comply with ethical recommendations of the University of Witwatersrand and of the United Nations Multi-Country Study on Men and Violence. ${ }^{40}$ Prospective participants were informed that they do not have to participate in the trial unless they are happy with the trial procedures and understand what the trial is about. All participants were told that participation is voluntary, that they may withdraw at any stage, skip any question in the research and that there are no adverse effects should they decide not to participate. For the success of the project, we require all research participants to agree in principle to multiple interviews (ie, baseline, 12 months and 24 months) - although they may change their mind.

The participant information leaflets and consent forms are written in simple English, however to enhance understanding, the explanation and discussion may be in isiZulu, Sepedi, Tsonga or English depending on the participant's language preference. A researcher was present throughout the informed consent process and clarified any questions the participants were not clear about. Once they are fully informed about the study, they were asked to sign informed consent for the interview. Participants also are asked for written informed consent to have their interview digitally recorded. Anonymity is important because of the sensitive nature of some of the questions. All questionnaires are identified by study identification numbers that are directly assigned by the electronic data system. Participants are reimbursed for their time to participate in the study. An amount of R50 (approximately US\$3.50) was paid to participants at the baseline data collection.

Participants who report sexual violence perpetrated against either partners or non-partners are not asked the age of the woman. South African law requires mandatory reporting of violence perpetrated against a minor (under the age of 18 years). Participants were informed during the consent process that if they disclose that they have perpetrated VAW to the research assistant that the incident may need to be reported to the police. However, since research assistants do not actively ask any of the questionnaire items, the opportunities for participants to disclose illegal behaviours are reduced.

Should the intervention or research teams become aware of any women who have experienced partner or non-partner violence, a protocol is in place to refer women to local organisations that provide counselling and support for survivors. Should any men disclose personal experiences of violence or be supporting family members who have experienced violence similar referrals for counselling and support are made. The list of referral organisations was developed in consultation with members of the $\mathrm{CAB}$ to ensure that services are accessible by community members and actively able to take new clients.

\section{Adverse reporting}

In social and behavioural trials, it is important for researchers to 'go beyond' typical medical reporting (which includes only physical health outcomes like hospitalisation or mortality) and report on social harms. We will take the most conservative approach to reporting and include all potential social harms within our definition of AEs, as noted in italics. AEs are any untoward medical or social occurrence that may present during intervention but which does not necessarily have a causal relationship with this project. AEs include risks to participant or fieldworker safety and any breach of confidentiality. Serious AEs (SAEs) are any untoward medical or social occurrence that results in death or significant disability or incapacity (including incarceration). SAEs may also include civil unrest or natural disaster in a study site that has the potential to put at serious risk the interviewers, participants or data quality. All reporting is following protocol established by the University of Witwatersrand Ethics Committee.

\section{Data monitoring}

A data monitoring committee was not established for this trial since the intervention is implemented at the community level, limiting the ability of an outside body to determine a statistical or ethical rationale for stopping rules. The CAB does serve as a local accountability mechanism for data at baseline and endline. The scientific steering committee of What Works to Prevent Violence has access to all study protocols and conducts annual checks of data quality and scientific progress. However, unlike some cluster randomised trials, there is not a dedicated data monitoring committee, which may be viewed as a weakness of this study design.

\section{Dissemination}

The final trial dataset will be made accessible to trial investigators for a period of 5 years. During this time, scholarly dissemination will take place through peer-reviewed journals and community dissemination will occur through a series of workshops with key community stakeholders and members of the network of non-governmental organisations working in the area to address VAW and children. After 5 years, the trial dataset will be made available to other researchers through an online portal managed by the What Works to Prevent Violence programme.

\section{DISCUSSION}

There are many well-documented efforts to reduce VAW from industrialised countries in North America and Europe ${ }^{43} 44$ with limited evidence from low-income and 
middle-income country settings. Many of the evaluated interventions have focused on the response to VAW rather than on primary prevention. Interventions that address the response to VAW have shown impact on physical and mental health outcomes for women, but there is limited evidence that these interventions reduce violence.

There are limitations inherent to the design of the C-RCT. Primary and secondary outcomes are self-reported which could result in either over-reporting or under-reporting. It is possible that the self-reporting bias will be different in intervention and control clusters. Men in the intervention clusters may under-report use of VAW at follow-up due to exposure to the intervention and social desirability bias. A strength of the study is that we are collecting longitudinal qualitative data through the process evaluation which will allow for triangulation between different components of the study. However, we are not collecting data from female partners of male participants, due to the safety risks associated with such dyadic data collection. Therefore, like many studies in the violence field, the primary trial outcome will be based on self-reported measures.

The risk of contamination is high due to the close physical proximity of the clusters and the nature of the intervention, which includes community mobilisation and advocacy elements. In addition, our formative research has revealed that men's movement within the 'township' is fairly common, which means that over the 2 years of follow-up, men may move from an intervention to a control cluster or vice versa. Our analysis will be based on intention-to-treat to address the movement of men across clusters. We recruited participants and then randomised the clusters after baseline data collection. However, once the intervention activities commence, it will no longer be possible to blind participants or implementers to which arm of the cluster they have been randomised. As with all longitudinal studies, lost to follow-up is a potential study limitation. Efforts will be made to collect different types of contact information of participants as well as up to four close friends or family members. The 2 years of follow-up data collection may be too short to measure an effect of the intervention since the recent use of violence is asked for the past 12 months. However, we believe that if the intervention is delivered as planned that changes in the primary outcome are possible.

The Sonke CHANGE trial will contribute to the limited body of evidence from low-income and middle-income countries of What Works to Prevent VAW and Girls. It will contribute to a growing set of studies that have explored whether gender transformative approaches work to reduce VAW. The trial together with the process evaluation will provide insight on whether the hypothesised pathways to change are relevant and appropriate. Moreover, we will gain insight into how change happens, if at all. Identifying and measuring interventions for addressing men's use of VAW is essential if we are to ensure the health and well-being of women, children and men themselves.
Acknowledgements The authors acknowledge the What Works to Prevent Violence against Women and Girls programme, and UKAID for providing the funding for this trial.

Contributors NJC: conceptualised the study together with AH and AP, and wrote the first draft of the manuscript. AH: conceptualised the study together with NJC and AP, and made substantial contributions to the writing of the manuscript. RSM: refined the process evaluation and contributed to the description of the process evaluation in the manuscript. DP, DR, AP and AA: developed and refined the Sonke intervention which the C-RCT is evaluating and commented on the manuscript.

Funding The Sonke CHANGE Trial is funded by the What Works to Prevent Violence against Women and Girls programme, South African Medical Research Council and UKAID; contact person: Professor Rachel Jewkes, rachel.jewkes@mrc.ac.za

Competing interests None declared.

Patient consent Obtained.

Ethics approval University of the Witwatersrand Human Research Ethics Committee.

Provenance and peer review Not commissioned; externally peer reviewed.

Open Access This is an Open Access article distributed in accordance with the Creative Commons Attribution Non Commercial (CC BY-NC 4.0) license, which permits others to distribute, remix, adapt, build upon this work non-commercially, and license their derivative works on different terms, provided the original work is properly cited and the use is non-commercial. See: http://creativecommons.org/ licenses/by-nc/4.0/

(C) Article author(s) (or their employer(s) unless otherwise stated in the text of the article) 2018. All rights reserved. No commercial use is permitted unless otherwise expressly granted.

\section{REFERENCES}

1. World Health Organization. Global and regional estimates of violence against women: prevalence and health effects of intimate partner violence and non-partner sexual violence. Geneva: World Health Organization, 2013.

2. Devries KM, Mak JY, García-Moreno C, et al. Global health. The global prevalence of intimate partner violence against women. Science 2013;340:1527-8.

3. Dunkle KL, Jewkes RK, Nduna M, et al. Perpetration of partner violence and HIV risk behaviour among young men in the rural Eastern Cape, South Africa. AIDS 2006;20:2107-14.

4. Jewkes R, Sikweyiya Y, Morrell R, et al. Gender inequitable masculinity and sexual entitlement in rape perpetration South Africa: findings of a cross-sectional study. PLoS One 2011;6:e29590.

5. Barker G, Contreras JM, Heilman B, et al. Evolving men: initial results from the International Men and Gender Equality Survey (IMAGES). Washington, DC: International Centerfor Research on Women (ICRW) and Instituto Promundo, 2011.

6. Fulu E, Warner X, Miedema S, et al. Why do some men use violence against women and how can we prevent it? Findings from the UN Multi-country study on men and violene in Asia and the Pacific. Bangkok: UNDP, UNFPA, UN Women, UNV, 2013.

7. Jewkes R, Morrell R. Gender and sexuality: emerging perspectives from the heterosexual epidemic in South Africa and implications for HIV risk and prevention. J Int AIDS Soc 2010;13:6.

8. Santana MC, Raj A, Decker MR, et al. Masculine gender roles associated with increased sexual risk and intimate partner violence perpetration among young adult men. $J$ Urban Health 2006;83:575-85.

9. Mankowski ES, Maton KI. A community psychology of men and masculinity: historical and conceptual review. Am J Community Psychol 2010;45:73-86.

10. Jewkes R, Nduna M, Levin J, et al. Impact of stepping stones on incidence of HIV and HSV-2 and sexual behaviour in rural South Africa: cluster randomised controlled trial. BMJ 2008;337:a506.

11. Kalichman SC, Simbayi LC, Cloete A, et al. Integrated gender-based violence and HIV Risk reduction intervention for South African men: results of a quasi-experimental field trial. Prev Sci 2009;10:260-9.

12. Pronyk PM, Hargreaves JR, Kim JC, et al. Effect of a structural intervention for the prevention of intimate-partner violence and HIV in rural South Africa: a cluster randomised trial. Lancet 2006;368:1973-83.

13. van den Berg W, Hendricks L, Hatcher A, et al. 'One Man Can': shifts in fatherhood beliefs and parenting practices following a gender- 
transformative programme in Eastern Cape, South Africa. Gend Dev 2013;21:111-25.

14. Peacock RD, Reneke PA, Forney GP. CFAST-consolidated model of fire growth and smoke transport (version 6) user's guide. Citeseer, 2013.

15. Connell RW. Gender and power: Society, the person and sexual politics. John Wiley \& Sons, 2014

16. Shefer T. From boys to men: Social constructions of masculinity in contemporary society. Juta and Company Ltd, 2007.

17. What Works to Prevent Violence. Summary of the Evidence and Research Agenda for What Works: a Global Programme to Prevent Violence against Women and Girls. London: UKAid, 2014.

18. Dworkin SL, Hatcher AM, Colvin C, et al. Impact of a gendertransformative HIV and antiviolence program on gender ideologies and masculinities in two rural, South African communities. Men Masc 2013;16:181-202.

19. Hatcher AM, Colvin CJ, Ndlovu N, et al. Intimate partner violence among rural South African men: alcohol use, sexual decision-making, and partner communication. Cult Health Sex 2014;16:1023-39.

20. García-Moreno C, Amin A. The sustainable development goals, violence and women's and children's health. Bull World Health Organ 2016;94:396-7.

21. Mahajan S. Economic of South African townships: special focus on Diepsloot. Washington DC: World Bank Group, 2014.

22. Jewkes R, Dartnall E, Sikweyiya Y. Ethical and Safety Recommendations for Research on the Perpetration of Sexual Violence. Pretoria, South Africa: Sexual Violence Research Initiative, Medical Research Council, 2012.

23. Courtenay WH. Constructions of masculinity and their influence on men's well-being: a theory of gender and health. Soc Sci Med 2000;50:1385-401.

24. Messner MA. Politics of masculinities: Men in movements. Sage Publications, Inc, 1997.

25. Freire P. Pedagogy of the Oppressed. New York: Continuum, 1970.

26. Freire P. Education for Critical Consciousness. New York: Continuum, 1973.

27. Jewkes R, Sikweyiya $Y$, Morrell R, et al. The relationship between intimate partner violence, rape and HIV amongst South African men: a cross-sectional study. PLoS One 2011;6:e24256.

28. Tsai AC, Tomlinson M, Comulada WS, et al. Intimate partner violence and depression symptom severity among south african women during pregnancy and postpartum: Population-based prospective cohort study. PLoS Med 2016;13:e1001943.

29. Saunders JB, Aasland OG, Babor TF, et al. Development of the alcohol use disorders identification Test (AUDIT): WHO Collaborative project on early detection of persons with harmful alcohol consumption-II. Addiction 1993;88:791-804.
30. Pulerwitz J, Barker G. Measuring attitudes towards gender norms among young men in Brazil: Development and psychometric evaluation of the GEM scale. Men and Masculinities 2008;10:322-38.

31. MRC. War at Home: Preliminary findings of the Gauteng Gender Violence Prevalence Study Johannesburg. Gender Links and the Medical Research Council, 2010. http://www.mrc.ac.za/gender/ gbvthewar.pdf

32. Pulerwitz J, Amaro H, De Jong W, et al. Relationship power, condom use and HIV risk among women in the USA. AIDS Care 2002;14:789-800.

33. Dunkle KL, Jewkes RK, Brown HC, et al. Gender-based violence, relationship power, and risk of HIV infection in women attending antenatal clinics in South Africa. Lancet 2004;363:1415-21.

34. Hatcher AM, Tsai AC, Kumbakumba E, et al. Sexual relationship power and depression among HIV-infected women in Rural Uganda. PLoS One 2012;7:e49821.

35. Straus MA, Hamby SL, Finkelhor D, et al. Identification of child maltreatment with the Parent-Child Conflict Tactics Scales: development and psychometric data for a national sample of American parents. Child Abuse Negl 1998;22:249-70.

36. Radloff LS. The CES-D scale a self-report depression scale for research in the general population. Appl Psychol Meas 1977;1:385-401.

37. Mollica RF, Caspi-Yavin Y, Bollini P, et al. The Harvard Trauma Questionnaire. Validating a cross-cultural instrument for measuring torture, trauma, and posttraumatic stress disorder in Indochinese refugees. J Nerv Ment Dis 1992;180:111-6.

38. Deitchler M, Ballard T, Swindale A, et al. Validation of a measure of household hunger for cross-cultural use. Washington, DC: Food and Nurtrition Technical Assistance II Project (FANTA-2), Acedemy for Educational Development, 2010.

39. Hayes RJ, Moulton LH. Cluster Randomised Trials. CRC Press, 2009

40. Fulu E, Jewkes R. Replicating the UN Multi-Country Study on men and violence: understanding why some men use violence against women and how we can prevent it. Bangkok, Thailand: Partners for Prevention, 2014.

41. Petersen ML, Sinisi SE, van der Laan MJ. Estimation of direct causal effects. Epidemiology 2006;17:276-84.

42. Patton MQ. Qualitative research. Wiley Online Library, 2005.

43. Wathen $\mathrm{CN}$, MacMillan HL. Interventions for violence against women: scientific review. JAMA 2003;289:589-600.

44. Ramsay J, Rivas C, Feder G. Interventions to reduce violence and promote the physical and psychosocial well-being of women who experience partner violence: a systematic review of controlled evaluations: Final report: Barts and the London. Queen Mary's School of Medicine and Dentistry, 2005. 